\title{
Reparación de defecto complejo de pared abdominal con colgajo una buena alternativa quirúrgica
}

\author{
Repair of a complex abdominal wall defect with anterolateral thigh flap: a good surgical \\ alternative
}

\author{
Julio Palacios-Juárez ${ }^{*}$, Jesús Morales-Maza², Marco A. Rendón-Medina ${ }^{3}$, Moisés Ramírez-Ramírez, \\ Jorge H. Rodríguez-Quintero ${ }^{2}$ y Ludivina A. Cortés-Martínez ${ }^{5}$ \\ ${ }^{1}$ Departamento de Cirugía Plástica y Reconstructiva, Hospital Regional de Alta Especialidad de Ixtapaluca, Estado de México; ${ }^{2}$ Servicio de Cirugía, \\ Instituto Nacional de Ciencias Médicas y Nutrición Salvador Zubirán, Ciudad de México; ${ }^{3}$ Servicio de Cirugía, Hospital Regional de Ixtapaluca, \\ Estado de México; ${ }^{4}$ Servicio de Cirugía Laparoscópica Avanzada, Hospital Regional de Alta Especialidad de Ixtapaluca, Estado de México; ${ }^{5}$ Servicio \\ de Anestesiología, Hospital Ángeles Lomas, Ciudad de México. México
}

\begin{abstract}
Resumen
Introducción: La reconstrucción de la pared abdominal con grandes defectos suele representar un desafío quirúrgico, sobre todo cuando los defectos son recurrentes y tienen un gran tamaño que dificulta la utilización de tejidos adyacentes para un cierre adecuado. La pared abdominal anterior se divide en tres regiones para su reconstrucción; superior, media e inferior. De acuerdo con cada región se han descrito diferentes técnicas de reconstrucción de pared, como la separación de componentes musculoaponeuróticos de la vaina de los rectos abdominales, el colgajo del músculo recto abdominal con o sin isla cutánea, el colgajo dependiente del músculo dorsal ancho y colgajos musculares o músculocutáneos del muslo para reconstruir el tercio inferior del abdomen, Ilamado colgajo anterolateral de muslo (ALT, por sus siglas en inglés). El ALT se ha convertido en una buena alternativa para los grandes defectos recurrentes en cualquiera de los tercios debido a su gran versatilidad. Caso clínico: Presentamos el caso de un paciente de 50 años con un defecto de pared abdominal, pérdida de dominio y exposición de material protésico (malla). Tenía el antecedente quirúrgico de colecistectomía abierta, necrosectomía por pancreatitis aguda con manejo de abdomen abierto (incisión en línea media supra-infraumbilical) e intento de reparación del defecto abdominal en dos ocasiones con colocación de malla (que se encontraba expuesta). El defecto existente se desmanteló y resecó en bloque desde el interior de la pared abdominal, dejando bordes aponeuróticos sanos, quitando todo el tejido cicatricial, la malla y los bordes cutáneos enfermos. Se realizó la reconstrucción de la pared abdominal con ALT con un componente de fascia lata con anastomosis a vasos epigástricos superiores, de manera exitosa. Actualmente no hay evidencia de recurrencia del defecto herniario tras 1 año de seguimiento. Discusión/conclusión: El objetivo de este trabajo es describir el ALT con componente de fascia lata con anastomosis a los vasos epigástricos superiores como una buena alternativa para reconstruir defectos de pared complejos del tercio superior del abdomen.
\end{abstract}

Palabras Clave: Colgajo anterolateral de muslo. Hernia compleja. Reconstrucción de pared abdominal. 


\section{Abstract}

Introduction: Reconstruction of the abdominal wall with major defects usually represents a surgical challenge, especially in cases where the defects are recurrent and have a large size that avoids the use of adjacent tissues for an adequate closure. According to each region the abdomen topography is divided into three regions: upper, middle and lower. Several reconstructive alternatives have been described according to the affected area of the abdomen that include the separation of the muscularis aponeurotic components of the abdominal rectus sheath, the flap of the rectus abdominus muscle with or without cutaneous island, the flap dependent on the dorsal muscle and muscular or musculocutaneous thigh flaps to reconstruct the lower area of the abdomen which is called anterolateral thigh (ALT) flap. The ALT flap has become the best option for large recurrent defects in any of the thirds due to its great versatility. Case report: We present the case of a 50-year-old patient with an abdominal wall defect, loss of domain and exposure of prosthetic material. Patient had a surgical history of open cholecystectomy, necrosectomy due to acute pancreatitis with open abdomen management and attempted repair of the abdominal defect twice with mesh placement. The abdominal wall was reconstructed with an ALT free flap with a fascia lata component with anastomosis to superior epigastric vessels in a successful manner. Nowadays patient remains without evidence of recurrence of the hernia at 1 year follow-up. Discussion/conclusion: The aim of this paper is to illustrate the ALT flap with a fascia lata component anastomosed to the superior epigastric vessels as a good option to reconstruct complex defects of the upper third of the abdomen.

Key Words: Anterolateral thigh flap. Complex abdominal hernia. Abdominal wall reconstruction.

\section{Introducción}

Los grandes defectos de la pared abdominal suelen ser un desafío quirúrgico, por lo que se han descrito varias alternativas reconstructivas entre las que se incluyen la separación de componentes, colgajos libres y colgajos pediculados con o sin colocación de malla; la elección de la técnica depende del tamaño del defecto, de su ubicación (tercio superior, medio o inferior) y del grado de contaminación del defecto herniario ${ }^{1-3}$.

Tradicionalmente, la reconstrucción de la pared abdominal se ha realizado con tejido adyacente. El tercio superior se reconstruye con colgajo dorsal ancho y recto abdominal con o sin isla cutánea. La reparación del tercio medio abdominal se realiza con separación de componentes musculoaponeuróticos de la vaina de los rectos abdominales, con liberación del músculo transverso abdominal, recto abdominal u oblicuo externo. En el tercio inferior se utilizan colgajos musculares o musculocutáneos provenientes del muslo, de forma pediculada (vasto lateral, gracilis y tensor de la fascia lata). Los colgajos libres son los siguientes: anterolateral del muslo (ALT, por sus siglas en inglés), anteromedial y los antes descritos, pero de forma libre. Los colgajos libres se han posicionado en las últimas dos décadas como opciones de primer orden para reparar grandes defectos abdominales ${ }^{4,5}$.

La tasa de recurrencia cuando se realiza la reconstrucción de la pared abdominal mediante cierre primario va del 12 al $54 \%$, y cuando se coloca malla protésica es del 2 al $36 \%$ 6,7. La recurrencia al utilizar colgajos pediculados o libres es desconocida, y tampoco se conoce al emplear sustitutos de dermis ${ }^{3}$.

Para abordar la reconstrucción de la pared abdominal se debe tomar en cuenta la calidad de las estructuras anatómicas afectadas (piel o fascia). Las opciones reconstructivas en la superficie de la piel incluyen el cierre primario, la expansión tisular y la remodelación de la piel con avance del tejido local o colgajos distantes (colgajos pediculados o libres) ${ }^{8-10}$. La reconstrucción fascial puede hacerse con separación de componentes musculoaponeuróticos de la vaina de los rectos abdominales, material protésico (sintético o biológico) y otras alternativas experimentales, como el injerto dérmico profiláctico autólogo ${ }^{11}$.

Se han descrito muchos colgajos para la reconstrucción de la pared abdominal, pero el ALT se está convirtiendo en el colgajo libre ideal para reconstruir los distintos defectos de la pared abdominal debido a su gran versatilidad, ya que pueden incluirse componentes fasciales (fascia lata) e incluso musculares (vasto lateral) $)^{12-14}$.

Este trabajo tiene como objetivo ilustrar el caso de un paciente con hernia de pared abdominal grande y recurrente del tercio superior abdominal, con pérdida de dominio abdominal e infección del sitio quirúrgico, que fue reconstruido con un ALT libre con componente de fascia lata (ALT-FL) y anastomosis a los vasos epigástricos superiores, ya que existe poca experiencia de reconstrucción abdominal en este contexto. 


\section{Caso clínico}

Se trata de un paciente de sexo masculino, de 50 años, con antecedentes de diabetes tipo 2 de reciente diagnóstico, e historia de consumo moderado de alcohol y tabaquismo con un índice tabáquico de 20 paquetes/año. Hace 17 años se le realizó una colecistectomía abierta y presentó pancreatitis aguda necrosante, tratada con necrosectomía y manejo con abdomen abierto y estancia hospitalaria de 2 meses. Previamente se había intentado reparar el defecto abdominal en dos ocasiones por recurrencia, empleando material protésico en los dos intentos. Después el paciente desarrolló una hernia abdominal con exposición de la malla. En la exploración física se apreciaba un defecto aponeurótico de $19 \times 10 \mathrm{~cm}$ y un contenido herniario con dimensiones de $9 \times 8 \times$ $3 \mathrm{~cm}$, donde se encontraba expuesto el material protésico (Fig. 1).

Se realizó la reconstrucción de la pared abdominal posterior a la resección en bloque del complejo herniario-granulomatoso cicatricial con la malla expuesta. Dicha reconstrucción se realizó mediante un colgajo libre de la región anterolateral del muslo izquierdo, con un componente de fascia lata para dar mejor soporte a la reparación del defecto (Fig. 2). El paciente presentó buena evolución y fue dado de alta sin complicaciones luego de 6 días de estancia hospitalaria. A continuación, describimos la técnica quirúrgica empleada.

\section{Técnica quirúrgica}

La cirugía se realizó bajo anestesia general con el paciente en posición en decúbito supino. Se inició con la resección de la malla y del tejido no viable, así como con una disección cuidadosa del defecto de la pared preservando las estructuras anatómicas en la medida de lo posible y realizando lavado quirúrgico. Se realizó la cosecha del colgajo ALT-FL.

La planificación quirúrgica del colgajo libre se realizó en la pierna izquierda, como describen Song, et al. ${ }^{14}$, basándonos en la arteria perforante más constante, la cual irriga el territorio vascular del tercio medio del muslo en su superficie anterolateral. Dicha arteria perforante se localiza habitualmente en el punto medio de una línea vertical que conecta la espina ilíaca anterosuperior con el borde superior y lateral de la rótula. En un radio de $3 \mathrm{~cm}$ del punto antes descrito se realizó un rastreo mediante Doppler acústico para identificar el sitio exacto de emergencia de la arteria perforante,

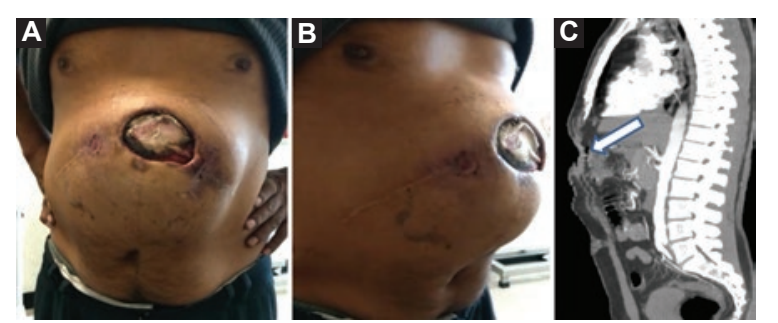

Figura 1. Evidencia de defecto de pared abdominal con exposición de material protésico. A: vista frontal del defecto abdominal. B: vista oblicua derecha del defecto. C: corte sagital izquierdo de tomografía de abdomen que muestra el defecto herniario.

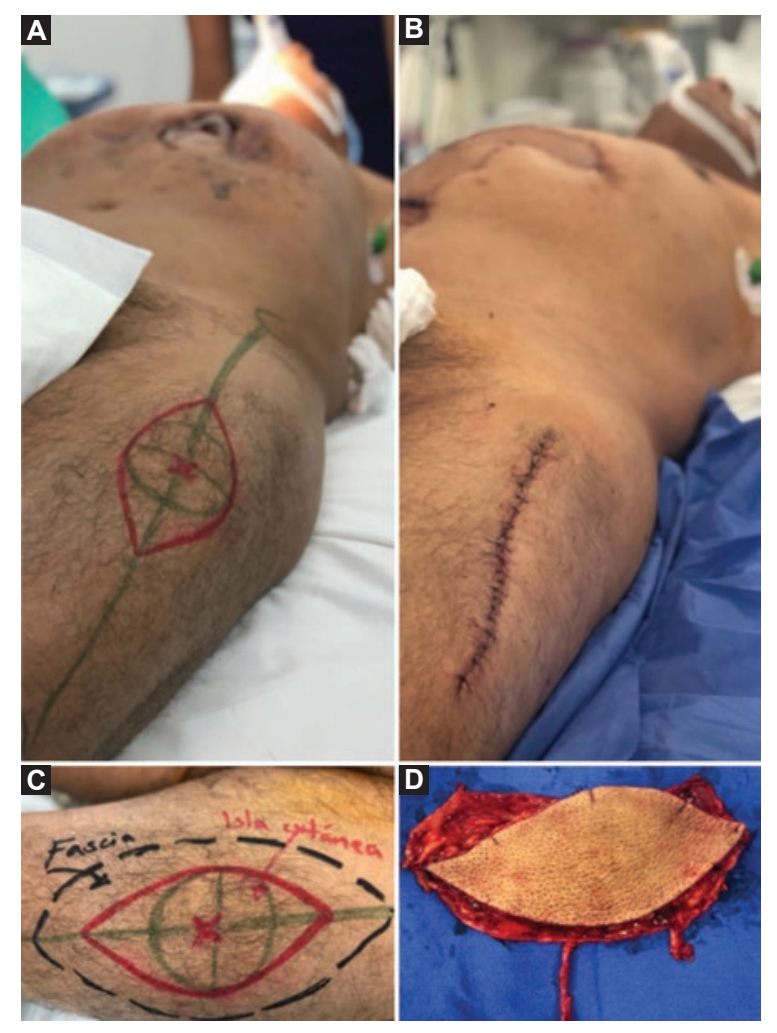

Figura 2. A: marcaje de isla cutánea del colgajo en el muslo izquierdo. B: herida resultante después de cosechar el colgajo (cierre directo). C: planeación del colgajo contemplando componente fascial con la arteria perforante identificada mediante Doppler acústico portátil. D: colgajo ALT-FL disecado con su pedículo en dirección inferior de la imagen.

y se marcó para poder diseñar el colgajo de las dimensiones necesarias dejando en el centro dicha perforante. Diseñamos un colgajo con componente de piel de $14 \times 8,5 \mathrm{~cm}$, con un área de $93,4 \mathrm{~cm}^{2}$, y componente de fascia lata más grande, de $24 \times 18 \mathrm{~cm}$, con un área de $339,3 \mathrm{~cm}^{2}$ (usando una ecuación de elipse, para una descripción del área más precisa) (Fig. 2).

La disección del colgajo se llevó a cabo en un plano subfascial, identificando la arteria perforante con sus venas concomitantes y disecando el pedículo de dicho colgajo (rama descendente de la arteria circunfleja 


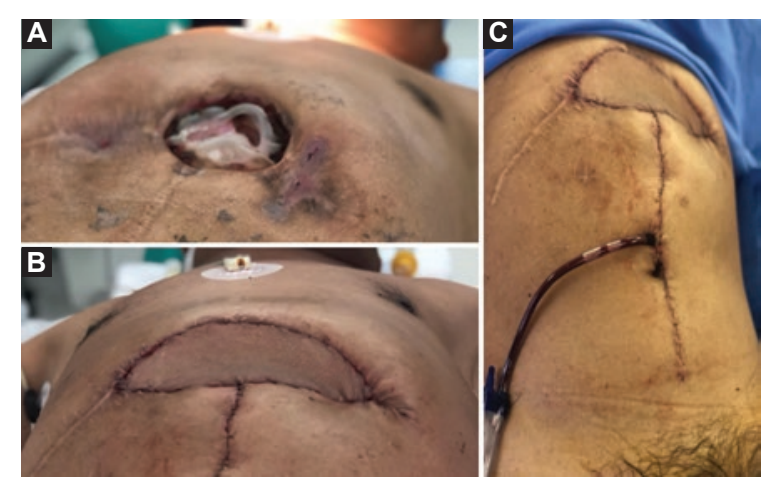

Figura 3. A: antes de la cirugía. B-C: después de la cirugía.
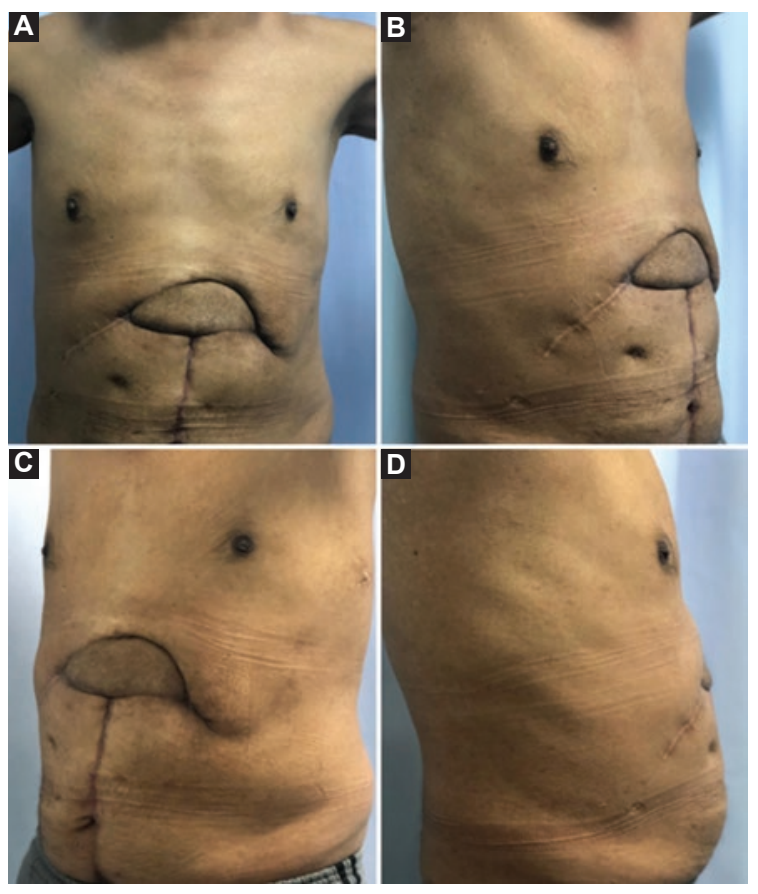

Figura 4. Seguimiento a 1 año del paciente sin evidencia de recurrencia del defecto herniario. A: vista frontal del abdomen. B: vista oblicua derecha del abdomen. C: vista oblicua izquierda. D: vista lateral derecha del abdomen, con buena continencia de la pared abdominal.

femoral lateral) hasta $12 \mathrm{~cm}$ de longitud, donde encontramos los vasos del calibre y del tamaño adecuados para su anastomosis con los vasos epigástricos superiores (arteria 2,5 $\mathrm{mm}$ y venas 3,5 $\mathrm{mm}$ de diámetro). El sitio donador del muslo izquierdo fue cubierto con un injerto de espesor parcial medio, tomado del mismo muslo. Se colocó el colgajo en el sitio a reparar, en el tercio superior del abdomen, y previa disección del pedículo receptor se realizó la anastomosis vascular con la ayuda de microscopio a la arteria epigástrica superior y las venas concomitantes, usando puntos separados de sutura microvascular 8-0. Una vez comprobado el adecuado flujo arterial al colgajo se cubrió el defecto abdominal, primeramente suturando el componente fascial del colgajo con los bordes aponeuróticos sanos que fueron disecados; dicha sutura se realizó con material absorbible (polidioxanona 2-0) realizando surgete continuo. A continuación se realizó el cierre del componente graso con material absorbible, y por último se cerró la piel con puntos separados. Se dejó un drenaje cerrado por 8 días, hasta que se obtuvo un gasto menor de $30 \mathrm{ml} \mathrm{y}$ fue retirado (Fig. 3).

El paciente fue dado de alta al sexto día de estancia hospitalaria, sin complicaciones. En el seguimiento a 1 semana presentó como complicación un seroma en el sitio quirúrgico abdominal, el cual se trató con un sistema de jeringa asistida por vacío que lo resolvió al cuarto día de seguimiento ${ }^{15}$. El paciente ha acudido a las citas de control con adecuada cicatrización, y a 1 año de seguimiento presenta buena continencia de la pared abdominal (Fig. 4).

\section{Discusión}

Presentamos el caso de un paciente con un defecto de pared abdominal en su tercio superior con pérdida de dominio, previamente operado en dos ocasiones con material protésico sin obtener un resultado satisfactorio, incluso con signos clínicos de infección, con exudado fétido y exposición de la malla. A partir de las opciones reconstructivas descritas elegimos la transferencia de colgajo ALT-FL para cerrar el defecto. El paciente presentaba una cicatriz tipo Kocher por una colecistectomía abierta y Chevron por una necrosectomía, lo que afectaba la calidad de la piel adyacente e impedía el uso de recto abdominal y dorsal ancho para reparar el defecto. No consideramos como opción la expansión tisular, ya que la exposición del material protésico podría contaminar el sitio de colocación del expansor y complicar aún más la reconstrucción.

Ting, et al ${ }^{12}$ reportaron el caso de un hombre de 60 años con un adenocarcinoma rectal y actividad metastásica en la pared abdominal anterior, con reconstrucción de un defecto de $20 \times 20 \mathrm{~cm}$ con un colgajo ALT pediculado con una rotación de $180^{\circ}$, colocándolo por debajo del recto femoral y del sartorio. En este caso no fue la opción óptima por el tamaño del defecto y la distancia del punto pivote de rotación, que no permitía alcanzar esas distancias.

Zhou, et $\mathrm{al}^{16}$ reportaron el caso de un paciente de sexo masculino, de 37 años, con un fibroma recurrente en la parte inferior del abdomen. La lesión medía $18 \times 16 \mathrm{~cm}$ después de la resección, y se reconstruyó un defecto de $22 \times 18 \mathrm{~cm}$ con una malla protésica (Proceed $^{\circledR}$, PCM, Ethicon, EE.UU.) y dos colgajos ALT pediculados. El defecto estaba en la pared abdominal 
inferior y era amplio, lo que explica por qué emplearon dos colgajos ALT pediculados o dos transferencias libres de tejido.

Un tema controversial es el uso de material protésico en heridas contaminadas. Está surgiendo evidencia reciente que reporta una morbilidad aceptable con el uso de material sintético en estos escenarios. Idealmente se ha propuesto el uso de materiales biológicos, como la dermis acelular porcina, pero el costo impide su uso de manera sistemática al menos en los países en vías de desarrollo como el nuestro'.

Carbonell y $\mathrm{Cobb}^{17}$ revisaron varios estudios que demostraban que el paradigma de no usar material protésico en campos contaminados podría estar fuera de lugar; sin embargo, aún faltan más estudios aleatorizados controlados para comprobar esta hipótesis.

\section{Conclusiones}

El colgajo anterolateral de muslo es una buena alternativa para reconstruir defectos complejos de la pared abdominal en cualquiera de sus tercios por sus características anatómicas, incluyendo el componente fascial que se puede agregar para dar mayor fuerza y soporte a la pared abdominal. Su versatilidad se debe a la constante ubicación de su pedículo, a la longitud del mismo y a su fácil disección cuando existe el entrenamiento para realizar estos colgajos. El colgajo puede disecarse muy delgado, delgado o compuesto con un componente de fascia lata o muscular (o ambos), dependiendo de las necesidades del defecto abdominal y de cada paciente. El inconveniente de este tipo de colgajos es que se requieren microscopio, suturas microquirúrgicas y personal bien entrenado en técnicas microquirúrgicas, que no siempre están disponibles en todos los centros hospitalarios de México.

\section{Conflicto de intereses}

Los autores declaran no tener ningún conflicto de interés.

\section{Responsabilidades éticas}

Protección de personas y animales. Los autores declaran que los procedimientos seguidos se conformaron a las normas éticas del comité de experimentación humana responsable y de acuerdo con la Asociación Médica Mundial y la Declaración de Helsinki.

Confidencialidad de los datos. Los autores declaran que han seguido los protocolos de su centro de trabajo sobre la publicación de datos de pacientes.

Derecho a la privacidad y consentimiento informado. Los autores han obtenido el consentimiento informado de los pacientes y/o sujetos referidos en el artículo. Este documento obra en poder del autor de correspondencia.

\section{Bibliografía}

1. Burger JW, Luijendijk RW, Hop WC, Halm JA, Verdaasdonk EG, Jeekel J. Long-term follow-up of a randomized controlled trial of suture versus mesh repair of incisional hernia. Ann Surg. 2004;240:578-85.

2. Mudge M, Hughes LE. Incisional hernia: a 10 year prospective study of incidence and attitudes. Br J Surg. 1985;72:70-1.

3. Hodgson NC, Malthaner RA, Ostbye T. The search for an ideal method of abdominal fascial closure: a meta- analysis. Ann Surg. 2000; 231:436-42.

4. Patel NG, Ratanshi I, Buchel EW. The best of abdominal wall reconstruction. Plast Reconstr Surg. 2018;141:113e-36e.

5. Ramirez OM, Ruas E, Dellon AL. "Components separation" method for closure of abdominal-wall defects: an anatomic and clinical study. Plast Reconstr Surg. 1990;86:519-26.

6. Paul A, Korenkov M, Peters S, Köhler L, Fischer S, Troidl H. Unacceptable results of the Mayo procedure for repair of abdominal incisional hernias. Eur J Surg. 1998;164:361-7.

7. Anthony T, Bergen PC, Kim LT, Henderson M, Fahey T, Rege RV, et al. Factors affecting recurrence following incisional herniorrhaphy. World $\mathrm{J}$ Surg. 2000;24:95-101.

8. Korenkov M, Sauerland S, Arndt M, Bograd L, Neugebauer EA, Troidl H. Randomized clinical trial of suture repair, polypropylene mesh or autodermal hernioplasty for incisional hernia. Br J Surg. 2002;89:50-6.

9. Rodriguez ED, Bluebond-Langner R, Silverman RP, Bochicchio G, Yao A, Manson PN, et al. Abdominal wall reconstruction following severe loss of domain: the R Adams Cowley Shock Trauma Center algorithm. Plast Reconstr Surg. 2007;120:669-80.

10. Martis G, Damjanovich L. Use of double-layer autologous dermal flap in the treatment of recurrent and/or infected incisional hernias: presentation of the surgical technique and the results of 1-year follow-up - a prospective, consecutive cohort study. Hernia. 2016;20:461-70.

11. Kim DY, Lee J, Kim JT, Chang HK, Moon S-H. Reconstruction of a large full-thickness abdominal wall defect with flow-through-based alt flaps: a case report. Microsurgery. 2019;39:85-90.

12. Ting J, Trotter D, Grinsell D. A pedicled anterolateral thigh (ALT) flap for reconstruction of the epigastrium. Case report. J Plast Reconstr Aesthetic Surg. 2010;63:e65-7.

13. Chang SH, Hsu TC, Su HC, Tung KY, Hsiao HT. Treatment of intractable enterocutaneous fistula with an island pedicled anterolateral thigh flap in Crohn's disease - case report. J Plast Reconstr Aesthetic Surg. 2010;63:1055-7

14. Song YG, Chen GZ, Song YL. The free thigh flap: a new free flap concept based on the septocutaneous artery. Br J Plast Surg. 1984;37:149-59.

15. Juárez JP, Azuara GA, Maza JM, Estrada JEF, Vega DC, Zamora MZ, et al. Vacuum Assisted Syringe System (V.A.S.S. Device): how to manufacture it step by step. SM J Surg. 2017;3:1015.

16. Zhou B, Zhou X, Li Z, Chen JY, Peng XW, Yang LC, et al. Reconstruction of the lower abdominal region using bilateral pedicled anterolateral thigh flaps combined with poly-surgical mesh. Medicine (Baltimore). 2015;94:e2375.

17. Carbonell AM, Cobb WS. Safety of prosthetic mesh hernia repair in contaminated fields. Surg Clin North Am. 2013;93:1227-39. 